\title{
GENERALIZED HERGLOTZ WAVE FUNCTIONS FOR MODELLING WIRELESS NEARFIELD MULTIPATH SCATTERING ENVIRONMENTS
}

\author{
Rodney A. Kennedy, Thushara D. Abhayapala, Tony. S. Pollock \\ National ICT Australia \\ Department of Telecommunications Engineering, RSISE \\ Institute for Advanced Studies \\ The Australian National University, Canberra 0200 Australia
}

\begin{abstract}
.
We develop a general mathematical model for nearfield multipath scattering as a basis for studying the spatial limits imposed on multi-antenna wireless communication systems. This model generalizes the Herglotz Wave Function, which is an important tool in the study of inverse scattering problems, to a form where the scatterers can be nearfield. This permits the development of the most general form of spatial correlation which is known to constrain the capacity of wireless systems.
\end{abstract}

\section{INTRODUCTION}

A standard model for a multipath field in $\mathbb{R}^{3}$ is to represent it as a superposition of plane waves from discrete directions:

$$
u(\boldsymbol{x})=\sum_{p} a_{p} e^{i k \boldsymbol{x} \cdot \widehat{\boldsymbol{y}}_{p}},
$$

where the plane wave of index $p$ has complex amplitude $a_{p} \in \mathbb{C}$, the propagation direction is denoted by the unit vector $\widehat{\boldsymbol{y}}_{p}$ and $\boldsymbol{x} \cdot \boldsymbol{y}$ denotes the scalar product between vectors $x, y \in \mathbb{R}^{3}$.

A straightforward generalization of (1) is

$$
u(\boldsymbol{x})=\int_{\mathbb{S}^{2}} g(\widehat{y}) e^{i k x \cdot \widehat{y}} d s(\widehat{y}),
$$

where $\mathbb{S}^{2}$ denotes the unit sphere, $s(\widehat{y})$ is a surface element of $\mathbb{S}^{2}$ with unit normal $\widehat{y}$ and $g \in L^{1}\left(\mathbb{S}^{2}\right)$ is the kernel representing an angular amplitude distribution of farfield sources. Representation (2) implies any sources which contribute to the field are farfield ones.

When $g$ is in $L^{2}\left(\mathbb{S}^{2}\right)$, a stronger condition than $g \in L^{1}\left(\mathbb{S}^{2}\right)$, it is known as a Herglotz Kernel, and representation (2) is known as the Herglotz Wave Function $[1$, p.55]. Herglotz wave functions primarily find use in inverse scattering problems where it is natural to find a scattered field satisfy the condition $g \in L^{2}\left(\mathbb{S}^{2}\right)$.

In our context, we are interested in using representations of the form (2), or generalizations thereof, to model any physically realizable scattering environment. If we use (2) then we exclude fields which have components from nearfield sources, and if, further, $g \in L^{2}\left(\mathbb{S}^{2}\right)$ then we exclude some farfield sources as well including those of the form (1). There is a need for a more general representation than (2) which has been used to model spatial correlation in wireless communication scattering scenarios [2].

The natural arena for the Herglotz Kernel is the Hilbert Space $L^{2}\left(\mathbb{S}^{2}\right)$ with the natural inner product defined on $\mathbb{S}^{2}$. However, important classes of multipath fields cannot be directly associated

Research partially supported by the Australian Research Council. with kernels belonging to such spaces. Our objective is to rework (2) to find a integral representation for multipath where the kernel associated with practically important scattering fields can be associated with a Hilbert Space corresponding to finite energy signals. Then the full machinery of Hilbert Space theory can be brought to bear on the representation to transparently render its properties which to a large degree fully emulate the remarkable properties of the classical Herglotz wave function - yielding an infinite number of orthonormal representations for multipath fields and associated $L^{2}$ Fourier Series. As a by-product our theory subsumes and provides a modest simplification to the results given in [1].

\section{PROBLEM FORMULATION}

\subsection{Subspace Interpretation}

Fields of the form (1), (2) and Herglotz wave functions satisfy the homogeneous Helmholtz equation in $\mathbb{R}^{3}$, sometimes referred to as the reduced wave equation:

$$
\triangle u(\boldsymbol{x})+k^{2} u(\boldsymbol{x})=0,
$$

where $\triangle$ is the Laplacian, and $k$ is the wave number given by the real positive constant $k=2 \pi / \lambda$. Equation (3) holds in any region of space, a subset of $\mathbb{R}^{3}$, that excludes any sources. That all such solutions to (3) for a given source-free region define a linear subspace of functions follows from the linearity and homogeneity of (3). That is, if $u_{1}(\boldsymbol{x})$ and $u_{2}(\boldsymbol{x})$ are solutions to (3) in a region then $\alpha_{1} u_{1}(\boldsymbol{x})+\alpha_{2} u_{2}(\boldsymbol{x})$ is also a solution in the same region.

\subsection{Helmholtz Balls}

In (2) the kernel is defined on $\mathbb{S}^{2}$ and implicitly the sources may be regarded as being defined on an infinite sphere. Many of the special properties that can be attributed to the Herglotz wave function are actually a manifestation of the high degree of spherical symmetry and the implicit choice of spherically symmetric domains (albeit infinite domains). Hence in studying (3) we expect highly structured solutions whenever the region of interest is a ball

$$
\mathbb{B}_{R}^{3} \triangleq\left\{\boldsymbol{x} \in \mathbb{R}^{3}:\|\boldsymbol{x}\| \leq R\right\},
$$

where $R$ is the radius (usually finite but possibly infinite). We write the above problem more compactly as follows.

Key PRoblem. Determine the complete subspace of solutions $u \equiv u(x)$ to

$$
\triangle u+k^{2} u=0, \text { in } \mathbb{B}_{R}^{3} .
$$


Physically this means we wish to understand the complete set of valid wavefields for arbitrary source locations with the only condition that the sources are not in $\mathbb{B}_{R}^{3}$.

Our task will be to find a Hilbert Space formulation where we can study the geometry of solutions to (5) using complete orthonormal sequences and the like. In what follows we assume the reader is well-familiar with separable Hilbert Spaces $[3,4]$.

Given we are dealing with spherical regions, we often utilize a spherical coordinate system $(r, \theta, \phi)$ representing radius, colatitude and longitude. In a coordinate system independent form we have $\boldsymbol{x} \equiv(r, \theta, \phi),\|\boldsymbol{x}\|$ representing radius $r$, and $\widehat{\boldsymbol{x}}$ representing the direction $\theta$ and $\phi$.

\subsection{Modal Representation}

From [1], solutions to (5) can be represented as the following entire series expansion (which converges in the mean)

$$
u(\boldsymbol{x})=\sum_{m, n}\left(4 \pi i^{n} \alpha_{n}^{m}\right) j_{n}(k\|\boldsymbol{x}\|) \dot{Y}_{n}^{m}(\widehat{\boldsymbol{x}}),
$$

where $j_{n}(k\|x\|)$ is the spherical Bessel function of integer order $n, \alpha_{n}^{m}$ are complex coefficients, and $Y_{n}^{m}(\widehat{x}) \equiv Y_{n}^{m}(\theta, \phi)$ are the spherical harmonics (orthonormal on $\mathbb{S}^{2}$ ) given by

$$
Y_{n}^{m}(\theta, \phi) \triangleq \sqrt{\frac{2 n+1}{4 \pi} \frac{(n-|m|) !}{(n+|m|) !}} P_{n}^{|m|}(\cos \theta) e^{i m \phi},
$$

$P_{n}^{|m|}(\cdot)$ are the associated Legendre functions, and we have introduced the shorthand $\sum_{m, n} \triangleq \sum_{n=0}^{\infty} \sum_{m=-n}^{n}$.

We can interpret the countable set

$$
\left\{A_{n}^{m}(\boldsymbol{x})\right\} \triangleq\left\{i^{n} j_{n}(k\|\boldsymbol{x}\|) Y_{n}^{m}(\widehat{\boldsymbol{x}})\right\}
$$

as specifying a basis for the subspace of solutions to (5). These basis functions are orthogonal over any spherically symmetric region. The sense in which these can be normalized for any size ball is now determined.

Denote the volume element at $\boldsymbol{x}$ as

$$
d v(\boldsymbol{x}) \triangleq r^{2} \sin \theta d \phi d \theta d r
$$

and define the inner product ${ }^{1}$ and associated induced norm by

$$
\begin{aligned}
\langle f, g\rangle_{\mathbb{B}_{R}^{3}} & \triangleq \int_{\mathbb{B}_{R}^{3}} f(\boldsymbol{x}) \overline{g(\boldsymbol{x})} h_{R}(\|\boldsymbol{x}\|) d v(\boldsymbol{x}) \\
& \equiv \int_{0}^{R} h_{R}(r) r^{2} \int_{\mathbb{S}^{2}} f(r, \widehat{\boldsymbol{x}}) \overline{g(r, \widehat{\boldsymbol{x}})} d s(\widehat{\boldsymbol{x}}) d r
\end{aligned}
$$

and

$$
\|f\|_{\mathbb{B}_{R}^{3}}^{2} \triangleq\langle f, f\rangle_{\mathbf{B}_{R}^{3}} \equiv \int_{\mathbb{B}_{R}^{3}}|f(\boldsymbol{x})|^{2} h_{R}(\|\boldsymbol{x}\|) d v(\boldsymbol{x}),
$$

parametrized by a non-negative bounded real weighting function $h_{R}(r) \geq 0$ that may depend on either $R$ or $r$.

\footnotetext{
${ }^{1}$ This is actually a class of inner products given that the radial weighting term $h_{R}(r)$ can take different forms. We are assuming that $h_{R}(r)$ is chosen such that we do have an inner product, particularly we require that $\langle f, f\rangle_{\mathbb{B}^{3}}=0$ implies $f=0$ which is not obviously satisfied. To avoid clutter, ${ }^{R}$ for the inner product we suppress in the notation the explicit dependence on $h_{R}(r)$.
}

Then, with a suitable choice of weight function $h_{R}\langle r\rangle$, the set of functions satisfying $\|f\|_{\mathbf{B}_{R}^{3}}^{2}<\infty$ is a separable Hilbert Space, and solutions of (5) are a strict subspace. This subspace naturally forms a Hilbert Space.

. The $\left\{A_{n}^{m}(x)\right\}$ are orthogonal with respect to the inner product (10). This follows from the orthonormality of the spherical harmonics defined on the unit sphere, $\mathbb{S}^{2}$, which induces orthogonality of our wave expansion over $\mathbb{B}_{R}^{3}$ leading to

$$
\left\langle A_{n}^{m}, A_{q}^{p}\right\rangle_{\mathbb{B}_{R}^{3}}=\delta_{n q} \delta_{m p} \int_{0}^{R} h_{R}(r)\left[j_{n}(k r)\right]^{2} r^{2} d r
$$

where $\delta_{n q}$ and $\delta_{m p}$ are delta functions. Equation (12) shows orthogonality holds independently of the choice of the non-negative bounded real function $h_{R}(r) \geq 0$. However, $h_{R}(r)$ does influence the normalization, as we explore next.

\subsection{Complete Orthonormal Sequences}

Orthonormality as distinct from orthogonality is subtly connected with the size of the spherical region. Beginning with the unnormalized set (8), equation (12) indicates how to achieve orthonormality

$$
\left\{\varphi_{n ; R}^{m}\right\}_{m, n} \triangleq\left\{\frac{i^{n} j_{n}(k\|\boldsymbol{x}\|) Y_{n}^{m}(\widehat{x})}{\left(\int_{0}^{R} h_{R}(r)\left[j_{n}(k r)\right]^{2} r^{2} d r\right)^{1 / 2}}\right\}_{m, n}
$$

noting the normalizing factor is a function of both $n$ and $R$. Whence, given completeness, any solution $u(\boldsymbol{x})$ to our problem (5) has representation (in the sense of convergence in the mean of the induced norm)

$$
u=\sum_{m, n}\left\langle u, \varphi_{n ; R}^{m}\right\rangle_{\mathbb{B}_{R}^{3}} \varphi_{n ; R}^{m},
$$

where we have the Fourier coefficient

$$
\begin{aligned}
\beta_{n ; R}^{m} & \triangleq\left\langle u, \varphi_{n ; R}^{m}\right\rangle_{\mathbb{B}_{R}^{3}} \\
& =\frac{\int_{\mathbb{B}_{R}^{3}} u(\boldsymbol{x})(-i)^{n} j_{n}(k\|x\|) \overline{Y_{n}^{m}(\widehat{\boldsymbol{x}})} h_{R}(r) d v(\boldsymbol{x})}{\left(\int_{0}^{R} h_{R}(r)\left[j_{n}(k r)\right]^{2} r^{2} d r\right)^{1 / 2}}
\end{aligned}
$$

\subsection{Field Representations}

Direct comparison of (14) and (15) with (6) leads to

$$
\begin{aligned}
& \alpha_{n}^{m}=\frac{\left\langle u(\boldsymbol{x}), \varphi_{n ; R}^{m}(\boldsymbol{x})\right\rangle_{\mathbb{R}_{R}^{3}}}{4 \pi\left(\int_{0}^{R} h_{R}(r)\left[j_{n}(k r)\right]^{2} r^{2} d r\right)^{1 / 2}} \\
& =\frac{\int_{\mathbb{B}_{R}^{3}} u(\boldsymbol{x}) \overline{Y_{n}^{m}(\widehat{\boldsymbol{x}})}(-i)^{n} j_{n}(k\|\boldsymbol{x}\|) h_{R}(\|\boldsymbol{x}\|) d v(\boldsymbol{x})}{\int_{\mathbb{B}_{R}^{3}}\left[j_{n}(k\|\boldsymbol{x}\|)\right]^{2} h_{R}(\|\boldsymbol{x}\|) d v(\boldsymbol{x})} .
\end{aligned}
$$

Hence there are a plethora of ways to compute these coefficients based on different values of $R$ and different choices of $h_{R}(r)$. Here are some of the more interesting choices for $h_{R}(r)$ and the resulting expressions for $\alpha_{n}^{m}(16)$ :

EXAMPLE 1. If $h_{R}(\|\boldsymbol{x}\|)=\delta\left(\|\boldsymbol{x}\|-r_{0}\right)$ where $0<r_{0} \leq R$, and $j_{n}\left(k r_{0}\right) \neq 0$ then

$$
\alpha_{n}^{m}=\frac{1}{4 \pi i^{n} j_{n}\left(k r_{0}\right)} \int_{\mathbb{S}^{2}} u_{r_{0}}(\widehat{\boldsymbol{x}}) \overline{Y_{n}^{m}(\widehat{\boldsymbol{x}})} d s(\widehat{\boldsymbol{x}}),
$$

where $u_{r_{0}}(\widehat{x})$ is $u(x)$ restricted to the shell $\|x\|=r_{0}$. That is, provided $k$ is not a Dirichlet eigenvalue we can use the Spherical Harmonic Transform to determine $\alpha_{n}^{m}[1]$. 
EXAMPLE 2. If $h_{R}(\|x\|)=1$ then we have the most natural case for a finite sphere $(R<\infty)$

$$
\begin{aligned}
\alpha_{n}^{m} & =\frac{\int_{\mathbb{B}_{R}^{3}} u(\boldsymbol{x})(-i)^{n} j_{n}(k\|\boldsymbol{x}\|) \overline{Y_{n}^{m}(\widehat{\boldsymbol{x}})} d v(\boldsymbol{x})}{\int_{\mathbb{B}_{R}^{3}}\left[j_{n}(k\|\boldsymbol{x}\|)\right]^{2} d v(\boldsymbol{x})} \\
& =\frac{\int_{\mathbb{E}_{R}^{3}} u(\boldsymbol{x})(-i)^{n} j_{n}(k\|\boldsymbol{x}\|) \overline{Y_{n}^{m}(\widehat{\boldsymbol{x}})} d v(\boldsymbol{x})}{4 \pi \int_{0}^{R}\left[j_{n}(k r)\right]^{2} r^{2} d r}
\end{aligned}
$$

The denominator in (18) will be written

$$
\mathcal{J}_{n}(R) \triangleq \int_{0}^{R}\left[j_{n}(k r)\right]^{2} r^{2} d r
$$

for which there are known closed form expressions.

EXAMPLE 3. If $h_{R}(\|x\|)=1 / R$, and let $R \rightarrow \infty$ then we have the most natural case for the infinite sphere. In this case we use the expression

$$
\lim _{R \rightarrow \infty} \frac{1}{R} \int_{0}^{R} r^{2}\left[j_{n}(k r)\right]^{2} d r=\frac{1}{2 k^{2}}
$$

to simplify denominator of (16) to glean

$$
\alpha_{n}^{m}=\lim _{R \rightarrow \infty} \frac{(-i)^{n} k^{2}}{2 \pi R} \int_{\mathbb{P}_{R}^{3}} u(\boldsymbol{x}) j_{n}(k\|\boldsymbol{x}\|) \overline{Y_{n}^{m}(\widehat{\boldsymbol{x}})} d v(\boldsymbol{x}) .
$$

\section{ORTHONORMAL EXPANSIONS IN BALLS}

\subsection{Finite Sphere Case}

We now focus on the natural inner product, where $h_{R}(\|x\|)=1$. We show that there is a more general representation than (6) when dealing with spherical regions $\mathbb{B}_{R}^{3}$ of radius $R$.

THEOREM 1 (EXPANSION FOR FINITE SOURCE-FREE BALL). Consider the space of finite energy solutions to the homogeneous Helmholtz equation $\Delta u+k^{2} u=0$ in a spherical domain $\mathbb{B}_{R}^{3}$ of radius $R<\infty$. Then any bounded solution $u$ can be expressed in terms of an expansion

such that

$$
u(\boldsymbol{x})=\sum_{m, n} \beta_{n ; R}^{m} \frac{i^{n} j_{n}(k\|\boldsymbol{x}\|) Y_{n}^{m}(\widehat{\boldsymbol{x}})}{\left[\mathcal{J}_{n}(R)\right]^{1 / 2}}
$$

$$
\left\{\varphi_{n ; R}^{m}(\boldsymbol{x})\right\}_{m, n} \triangleq\left\{\frac{i^{n} j_{n}(k\|\boldsymbol{x}\|) Y_{n}^{m}(\widehat{\boldsymbol{x}})}{\left[\mathcal{J}_{n}(R)\right]^{1 / 2}}\right\}_{m, n}
$$

are orthonormal with respect to the inner product

$$
\langle f, g\rangle_{\mathbb{R}_{R}^{3}} \triangleq \int_{\mathbb{B}_{R}^{3}} f(\boldsymbol{x}) \overline{g(\boldsymbol{x})} d v(\boldsymbol{x}) .
$$

The Fourier Coefficients $\beta$ are given by

$$
\begin{aligned}
\beta_{n ; R}^{m} & =\left\langle u, \varphi_{n ; R}^{m}\right\rangle_{\mathbb{E}_{R}^{3}} \\
& =\int_{\mathbb{B}_{R}^{3}} u(\boldsymbol{x}) \frac{(-i)^{n} j_{n}(k\|\boldsymbol{x}\|) \overline{Y_{n}^{m}(\widehat{\boldsymbol{x}})}}{\left[\mathcal{J}_{n}(R)\right]^{1 / 2}} d v(\boldsymbol{x}) .
\end{aligned}
$$

and are square summable, that is, $\beta \in \ell^{2}$.

Proof. The orthonormality of (23) is a special case of (13) with $h_{R}(\|x\|)=1$. By Parseval

$$
\sum_{m, n}\left|\beta_{n ; R}^{m}\right|^{2}=\langle u, u\rangle_{\mathbb{B}_{R}^{3}}=\int_{\mathbf{B}_{R}^{3}}|u(\boldsymbol{x})|^{2} d v(\boldsymbol{x})<\infty
$$

by the finite energy of $u$, that is, $\left\|\beta_{R}\right\|_{\ell^{2}}^{2}=\|u\|_{\mathbb{B}_{R}^{3}}^{2}<\infty$.

\subsection{Infinite Sphere Case}

We now consider the case where the region is the whole space $\mathbb{R}^{3}$ which can be regarded as an infinite spherical volume. In this case we use $h_{R}(\|x\|)=1 / R$ and let $R \rightarrow \infty$. As we will see, the results indicate that the expansion in (6) is most naturally associated with the infinite sphere.

THEOREM 2 (EXPANSION FOR INFINITE SOURCE-FREE BALL). Let $u$ be any bounded solution to the homogeneous Helmholtz equation $\Delta u+k^{2} u=0$ in $\mathbb{R}^{3}$. Then $u$ can be expressed in terms of an expansion

$$
u(\boldsymbol{x})=\sum_{m, n} \beta_{n ; \infty}^{m} k \sqrt{2} i^{n} j_{n}(k\|\boldsymbol{x}\|) Y_{n}^{m}(\widehat{\boldsymbol{x}})
$$

such that

$$
\left\{\varphi_{n ; \infty}^{m}\right\}_{m, n} \triangleq\left\{k \sqrt{2} i^{n} j_{n}(k\|\boldsymbol{x}\|) Y_{n}^{m}(\widehat{\boldsymbol{x}})\right\}_{m, n}
$$

are orthonormal with respect to the natural inner product

$$
\langle f, g\rangle_{\mathbb{E}_{\infty}^{3}} \triangleq \lim _{R \rightarrow \infty} \frac{1}{R} \int_{\mathbb{B}_{R}^{3}} f(\boldsymbol{x}) \overline{g(\boldsymbol{x})} d v(\boldsymbol{x}),
$$

and the Fourier coefficients are given by

$$
\begin{aligned}
& \beta_{n ; \infty}^{m}=\left\langle u(\boldsymbol{x}), \varphi_{n ; \infty}^{m}(\boldsymbol{x})\right\rangle_{\mathbb{B}_{\infty}^{3}} \\
& =\lim _{R \rightarrow \infty} \frac{(-i)^{n} k \sqrt{2}}{R} \int_{\mathbb{B}_{R}^{3}} u(\boldsymbol{x}) j_{n}(k\|\boldsymbol{x}\|) \overline{Y_{n}^{m}(\widehat{\boldsymbol{x}})} d v(\boldsymbol{x})
\end{aligned}
$$

and are square summable, that is, $\beta_{\infty} \in \ell^{2}$.

Proof. The orthonormality of (28) is a special case of (13) with $h_{R}(\|x\|)=1 / R$ and letting $R \rightarrow \infty$.

Now we present a key representation result -- captured in Theorem 3.22 in [1] - for a class of solutions to the homogeneous Helmholtz equation.

Theorem 3 (Classical Herglotz Wave Function). Let $u$ be any bounded solution to the homogeneous Helmholtz equation $\Delta u+k^{2} u=0$ in $\mathbb{R}^{3}$ satisfying the growth condition

$$
\lim _{R \rightarrow \infty} \frac{1}{R} \int_{\mathbb{R}_{R}^{3}}|u(\boldsymbol{x})|^{2} d v(\boldsymbol{x})<\infty .
$$

Then we have the representation for $u$

$$
u(\boldsymbol{x})=\frac{k}{2 \pi \sqrt{2}} \int_{\mathbb{S}^{2}} b(\widehat{\boldsymbol{x}}) e^{i k \boldsymbol{x} \cdot \widehat{\boldsymbol{y}}} d s(\widehat{\boldsymbol{y}})
$$

where $b(\widehat{x})$ is, up to a constant, the Herglotz Kernel and can be expressed as the Inverse Spherical Harmonic Transform

$$
b(\widehat{x}) \triangleq \sum_{m, n} \beta_{n ; \infty}^{m} Y_{n}^{m}(\widehat{x}) \in L^{2}\left(\mathbb{S}^{2}\right)
$$

of the Fourier coefficients $\beta_{\infty} \in \ell^{2}$ given in Theorem 2 .

Proof. By Parseval

$$
\sum_{m, n}\left|\beta_{n ; \infty}^{m}\right|^{2}=\|u\|_{\mathbb{B}_{\infty}^{3}}^{2} \triangleq \lim _{R \rightarrow \infty} \frac{1}{R} \int_{\mathbb{E}_{R}^{3}}|u(\boldsymbol{x})|^{2} d v(\boldsymbol{x})
$$

which is finite by the growth condition (31). Hence $\beta_{\infty} \in \ell^{2}$ which implies that (33) is well-defined and in $L^{2}\left(\mathbb{S}^{2}\right)$. Then (33) can be inverted and this implies $\beta_{n}^{m}=\left\langle b, Y_{n}^{m}\right\rangle_{\mathbb{S}^{2}}$, leading to

$$
u=\sum_{m, n} \beta_{n}^{m} \varphi_{n ; \infty}^{m}=\sum_{m, n}\left\langle b, Y_{n}^{m}\right\rangle_{\mathbb{S}^{2}} \varphi_{n ; \infty}^{m}
$$


which equals

$$
\begin{aligned}
& u(\boldsymbol{x})= \sum_{m, n}\left(\int_{\mathrm{S}^{2}} b(\widehat{\boldsymbol{y}}) \overline{Y_{n}^{m}(\widehat{\boldsymbol{y}})} d s(\widehat{\boldsymbol{y}})\right) \times \\
& \sqrt{2} k i^{n} j_{n}(k\|\boldsymbol{x}\|) Y_{n}^{m}(\widehat{\boldsymbol{x}}) \\
&=\left(\frac{k}{2 \pi \sqrt{2}}\right) \int_{\mathbb{S}^{2}} b(\widehat{\boldsymbol{y}}) \times \\
&\{\underbrace{\left\{\pi \pi \sum_{m, n} i^{n} j_{n}(k\|\boldsymbol{x}\|) Y_{n}^{m}(\widehat{\boldsymbol{x}}) \overline{Y_{n}^{m}(\widehat{\boldsymbol{y}})}\right.}_{e^{i k \boldsymbol{x} \cdot \hat{\boldsymbol{y}}} \text { by the Jacobi-Anger Expansion [1] }}\} d s(\widehat{\boldsymbol{y}})
\end{aligned}
$$

Comment. Comparing (6), (21) and (30) we can see that

$$
\beta_{n ; \infty}^{m}=\left(\frac{2 \pi \sqrt{2}}{k}\right) \alpha_{n}^{m} \equiv(\lambda \sqrt{2}) \alpha_{n}^{m}, \quad \forall m, n .
$$

Clearly $\left\|\beta_{\infty}\right\|_{\ell^{2}}^{2}<\infty$ iff $\|\alpha\|_{\ell^{2}}^{2}<\infty$ where $\alpha \triangleq\left\{\alpha_{n}^{m}\right\}_{m, n}$.

\subsection{Generalized Herglotz Wave Functions}

As previously mentioned (32) is a Herglotz Wave Function, up to a constant. We now show how to generalize the classical Herglotz wave function to broaden its applicability and more transparently render its derivation and properties.

Let $\mathcal{H}$ be the separable Hilbert Space of solutions to the homogeneous Helmholtz equation $\Delta u+k^{2} u=0$ in $\mathbb{B}_{R}^{3} \triangleq\{\boldsymbol{x} \in$ $\left.\mathbb{R}^{3}:\|\boldsymbol{x}\| \leq R\right\}$, with inner product

$$
\langle f, g\rangle_{\mathbf{B}_{R}^{3}} \triangleq \int_{\mathbb{B}_{R}^{3}} f(\boldsymbol{x}) \overline{g(\boldsymbol{x})} d v(\boldsymbol{x}), \quad f, g \in \mathcal{H}
$$

Definition (GENERALIZED HERgLotz WAVE FUnCtion) Let $\varphi_{n ; R}^{m}$ be a complete orthonormal sequence in the separable Hilbert Space of solutions to the homogeneous Helmholtz equation given by $\triangle u+k^{2} u=0$ in $\mathbb{B}_{R}^{3} \triangleq\left\{\boldsymbol{x} \in \mathbb{R}^{3}:\|\boldsymbol{x}\| \leq R\right\}$ then

$$
v=\left\langle g, Y_{n}^{m}\right\rangle_{S^{2}} \varphi_{n ; R}^{m} .
$$

is a Generalized Herglotz Wave Function with Generalized Herglotz Kernel $g \in L^{2}\left(\mathbb{S}^{2}\right)$.

The proof of Theorem 3, particularly in (35), showed that this reduces to the classical Herglotz Wave Function. The essence of the Generalized Herglotz Wave Function is that a field can be represented by a $L^{2}$-function defined on the unit sphere $\mathbb{S}^{2}$ and this is captured by ( 39 ).

Fig. 1 indicates the relationships between the various representations. It shows that the classical Herglotz Wave Function can be viewed as a isomorphism between the space of square integrable functions defined on the unit sphere $\mathbb{S}^{2}$ and the space of wavefields generated by sources no closer than distance $R$ from the origin.

\section{APPLICATIONS}

\subsection{Spatial Correlation}

In [2] the expression (2) (which is a form of Herglotz Wave Function except that the kernel can be in $L^{1}\left(\mathbb{S}^{2}\right)$ and not only space $L^{2}\left(\mathbb{S}^{2}\right)$ ) was used to render in closed form an expression spatial correlation which subsumed a number of other explicit models which have appeared in the literature. The kernel can be associated with a farfield angular distribution of power from the scattering environment. With the generalized Herglotz Wave Function there are two further advantages: i) nearfield sources and scatterers can now be incorporated; and ii) the kernel can be chosen in $L^{2}\left(\mathbb{S}^{2}\right)$ from which the theory of Hilbert Spaces can be applied. We mention without proof, due to space limitations, that the generalized Herglotz Wave Function has a single layer and double layer potential interpretation defined on a spherical boundary. This potential function is the analogue of (2).

\subsection{Channel Representation}

The Generalized Herglotz Wave Function permits one to dispense with a potential complicated source and scatter geometry and replace it with a fully equivalent distribution defined on a spherical region. This decomposition of space and the model it implies will be presented in a future publication.

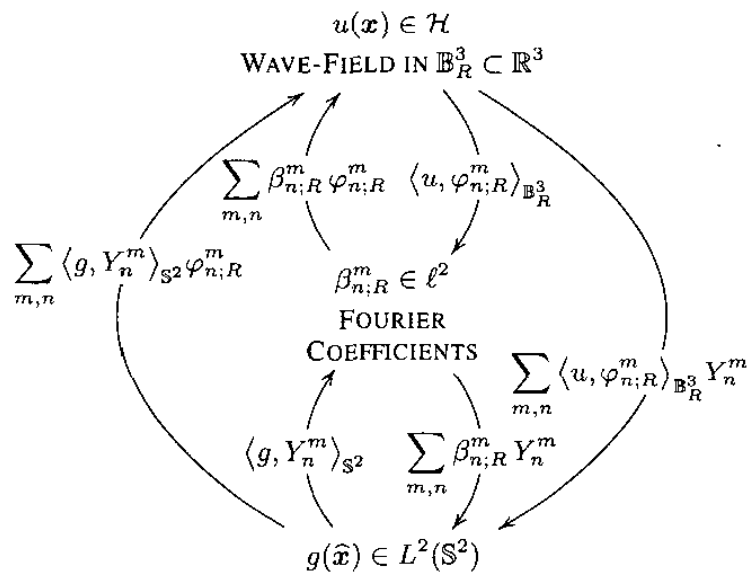

GENERALIZED HERGLOTZ

KERNEL ON $\mathbb{S}^{2}$

Fig. 1: Isomorphisms between the Wave Field $u(x)$ in $\mathcal{H}$, the Fourier Coefficients $\beta_{n ; R}^{m}$ in $\ell^{2}$ and the Herglotz Kernel $g(\widehat{x})$ in $L^{2}\left(\mathbb{S}^{2}\right)$. The mapping between the Generalized Herglotz Kernel and the Wave Field, $\sum_{m, n}\left\langle g, Y_{n}^{m}\right\rangle_{\mathcal{S}^{2}} \varphi_{n ; R}^{m}$ is the Generalized Herglotz Wave Function.

\section{REFERENCES}

[1] D. Colton and R. Kress, Inverse Acoustic and Electromagnetic Scattering Theory, Springer-Verlag, Berlin, Germany, second edition, 1998.

[2] P. D. Teal, T. D. Abhayapala, and R. A. Kennedy, "Spatial Correlation for General Distributions of Scatterers," in Proc. IEEE Int. Conf. Acoust., Speech, Signal Processing, ICASSP'2002, Orlando, Florida, May 2002, vol. 3, pp. 28332836.

[3] F. Riesz and B. Sz.-Nagy, Functional Analysis, Dover Publications Inc., New York, NY, second edition, 1990.

[4] L. Debnath and P. Mikusiński, Introduction to Hilbert Spaces with Applications, Academic Press, San Diego, CA, 1999. 